\title{
5 Research Square

\section{Energy Efficiency Optimization in Adaptive Massive MIMO Networks for 5G Applications Using Genetic Algorithm}

Ibrahim Salah ( $\square$ ibrahim.salah@nub.edu.eg )

Nahda University https://orcid.org/0000-0002-1616-6510

M. Mourad Mabrook

Nahda University

Kamel Hussein Rahouma

Nahda University

Aziza I. Hussein

Effat University

\section{Research Article}

Keywords: Adaptive antenna ,5G networks, Massive-MIMO, Energy efficiency optimization, Genetic Algorithm

Posted Date: September 17th, 2021

DOl: https://doi.org/10.21203/rs.3.rs-816195/v1

License: (9) This work is licensed under a Creative Commons Attribution 4.0 International License. Read Full License

Version of Record: A version of this preprint was published at Optical and Quantum Electronics on January 20th, 2022. See the published version at https://doi.org/10.1007/s11082-021-03507-5. 


\title{
Energy Efficiency Optimization in Adaptive Massive MIMO Networks for 5G Applications Using Genetic Algorithm
}

\author{
Ibrahim Salah $^{\text {a }}$, M. Mourad Mabrook ${ }^{\text {, }}$, Kamel Hussein Rahouma ${ }^{\mathrm{c}, \mathrm{d}}$, Aziza I. Hussein ${ }^{\mathrm{e}}$ \\ ${ }^{a}$ CCE department, faculty of Engineering, Nahda University, Beni-Suef, Egypt \\ ${ }^{b, a}$ Faculty of Navigation Science \& Space Technology, Beni-Suef University, Beni-Suef, Egypt \\ ${ }^{c}$ Department of Electrical Engineering, Faculty of Engineering, Minia University, Minia, Egypt \\ Faculty of Computer science, Nahda University, Beni-Suef, Egypt. \\ ${ }^{e}$ Electrical \& Computer Eng. Dept., Effat University, Jeddah, KSA
}

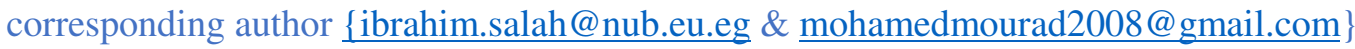

\begin{abstract}
Given that the exponential pace of growth in wireless traffic has continued for more than a century, wireless communication is one of the most influential innovations in recent years. Massive Multiple-Input Multiple-Output (M-MIMO) is a promising technology for meeting the world's exponential growth in mobile data traffic, particularly in $5 G$ networks. The most critical metrics in the massive MIMO scheme are Spectral Efficiency (SE) and Energy Efficiency (EE). For single-cell MMIMO uplink transmission, energy and spectral-efficiency trade-offs have to be estimated by optimizing the number of base station antennas versus the number of active users. This paper proposes an adaptive optimization technique focusing on maximizing Energy Efficiency at full spectral efficiency using a Genetic Algorithm (GA) optimizer. The number of active antennas is estimated according to the change in the number of active users based on the proposed GA scheme that optimizes the EE in the M-MIMO system. Simulation results show that the GA optimization technique achieved the maximum energy efficiency of the $5 G$ M-MIMO platform and the maximum efficiency in the trade-off process.
\end{abstract}

Keywords: Adaptive antenna ;5G networks; Massive-MIMO; Energy efficiency optimization; Genetic Algorithm.

\section{Introduction}

5G cellular networks aim to provide 1,000-fold capacity increases without higher additional costs on today's networks. Since network energy varies significantly during the day, $5 \mathrm{G}$ networks must be capable of changing power usage [1]. Massive MIMO is required to be the primary applicant technology capable of handling extremely high capacity. In a massive MIMO system, each Base Station (BS) employs hundreds of antennas to support tens of user equipment (UEs) on the same time-frequency resource [2].

Massive-MIMO relies on spatial multiplexing, which requires adequate channel information from the base station on both the up-link and down-link. Massive MIMO increases capability tenfold or more while also improving the radiated energy efficiency on a request by a factor of a hundred. Adjustment of Energy Efficiency (EE) plays a critical role in developing $5 \mathrm{G}$ massive MIMO systems.EE is estimated by the number of bits that can be realistically transmitted per joule as mentioned in equation (1), which has become a significant performance metric in wireless-communications $[3,4]$.

$E E=\frac{\text { Throughput }[\mathrm{bit} / \mathrm{s} / \mathrm{cell}]}{\text { Power Consumption }\left[\frac{\mathrm{W}}{\mathrm{cell}}\right]}$

Where the service quality and associated costs are compared to power consumption, it is now a metric for the network's bit-delivery reliability. From another side, Spectral Efficiency (SE) can be defined as "The average number of bits of information, that can reliably have transmitted under consideration over the channel [16]" as indicated in equation (2).

$S E=\frac{\text { Data rate or Throughput }(\mathrm{pbs})}{\text { channel Bandwidth }(\mathrm{Hz})}$

Massive (MIMO) systems, which employ many receiving antennas at each base station, significantly increase $\mathrm{SE}$ in the cell network [5], potentially improving EE over single-antenna techniques. The EE is reliant not only on the SE but also on Circuit Power (CP) utilization. The usage of large numbers of BS antennas in massive-MIMO will significantly increase 
the power consumption of radio-frequency (RF) circuits and digital signal processing (DSP), which has an apparent effect on the EE [6,7].

EE is one of the most important goals established for fifth-generation communications. As a result, many academics across the globe have been driving many studies on EE in communication systems. Various wireless methods have been proposed in the literature to enhance the EE of communication networks. When the SNR is low, Zhang et al. [8] developed an improved adaptive power allocation method that provides optimal EE. Due to greater SNRs, their suggested method outperforms the conventional power allocation technique.

MIMO methods may, according to Gong et al. [9], lead to fair trade between EE and SE. However, the radio frequency connectivity issue was disregarded. Liu et al. [10] proposed a resource distribution method for various users with varying power levels. Nevertheless, it leads to a great degree of difficulty. In addition, the authors of $[11,12]$ suggested a resource-allocation power-efficient method to resolve the energy consumption downlink problem in OFDM networks with a large number of base stations.

An adaptive massive MIMO optimization technique based on optimizing energy efficiency at full spectral efficiency is proposed in this paper. In order to achieve the optimum EE, the number of active antennas is adjusted based on the number of active-users in each network cell. Finally, Simulation results indicate that the proposed method has adjusted the number of active antennas based on changed active users in order to maximise $\mathrm{EE}$ of the $5 \mathrm{G}$ network Massive MIMO system.

The leftover of this paper is organized as follows: In Section 2, the Problem Formulation and system model are introduced. In Section 3, simulation analysis and results are considered. The conclusion of this paper is presented in Section 4.

\section{System design}

In this paper, EE and SE conditions and boundaries for data transmission are mainly being studied [13]. The proposed system model consists of a single-cell $[14,15]$ where one Massive-MIMO antenna BS simultaneously serving a set of User Equipment UE as shown in fig.1.

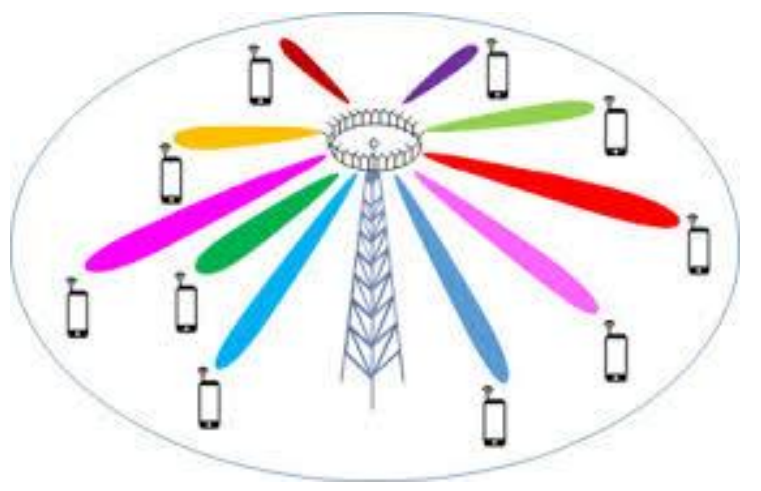

Fig.1. The Massive-MIMO Cell

\subsection{Impact of Multiple BS Antennas and Users}

While using Maximum Ratio (MR) combining with perfect channel information at the BS [16], the uplink (UL) SE of each UE is calculated according to equation (3)

$$
\mathrm{SE}_{0}=\log _{2}\left(1+\frac{M-1}{(K-1)+K \bar{\beta}+\frac{\sigma^{2}}{p \xi_{0}^{\mathrm{U}}}}\right)
$$

Where, $\mathrm{M}$ is the number of antennas per each base station, $\mathrm{K}$ is the number of user equipment's UEs, $p$ defines the transmit power, $\sigma^{2}$ means the noise power, and $\beta$ signify the average gain of channel of the active $\mathrm{UE}$ and it is assumed to be constant for all UEs in cell j. The corresponding EE of cell 0 is defined [17] according to equation (4)

$$
\mathrm{EE}_{0}=\frac{B K \mathrm{E}_{0}}{K\left(\frac{M-1}{\left.2^{\mathrm{S} \mathrm{E}_{0}-1}-K \bar{\beta}+1-K\right)^{-1} \nu_{0}+\mathrm{CP}_{0}}\right.}
$$

Where, B denotes the bandwidth, and $v_{0}$ is evaluated according to equation (5)

$$
v_{0}=\frac{\sigma^{2}}{\mu \beta_{0}^{0}}
$$

Where, $\boldsymbol{\mu}$ is a factor accounts for the Effective Transmit Power (ETP), $\quad 0<\boldsymbol{\mu}<1$.

The Circuit Power (CP) model consumed by single UE is estimated according to:

$$
C P_{0}=P_{F I X}+M P_{B S}
$$


Where, $\mathrm{P}_{\mathrm{FIX}}$ is the fixed power and $\mathrm{P}_{\mathrm{BS}}$ refers to the power utilized by the circuit components (e.g., DACs, ADCs, I/Q mixers, filters, Local Oscillator, and OFDM modulation/demodulation) in which they are needed for the operation of each BS antenna.

In order to calculate the additional Circuit Power (CP) consumed by all the active UEs [16], $\mathrm{CP}_{0}$ is estimated according to equation (7)

$C P_{0}=P_{F I X}+M P_{B S}+K P_{U E}$

Where, $\mathrm{P}_{\mathrm{UE}}$, refers to the power required by all circuit components (e.g., I/Q mixer, DAC, filter, and so forth) of each UE's single-antenna.

Derivative of equation (4), is obtained to get the Maximum Energy Efficiency (Max EE), [17] yields the expression:

$$
\begin{aligned}
& \max E E=\frac{d}{d S E 0} E E_{0} \\
& \max E E \approx \frac{e B}{(1+e)} \frac{\log _{2}\left(M P_{F I X}\right)}{P_{F I X}}
\end{aligned}
$$

Equation (8) proves that, the maximum EE enhances logarithmically with the number of antennas per base station (M) and has an almost linear decreasing function with increasing $\mathrm{P}_{\mathrm{FIX}}$.

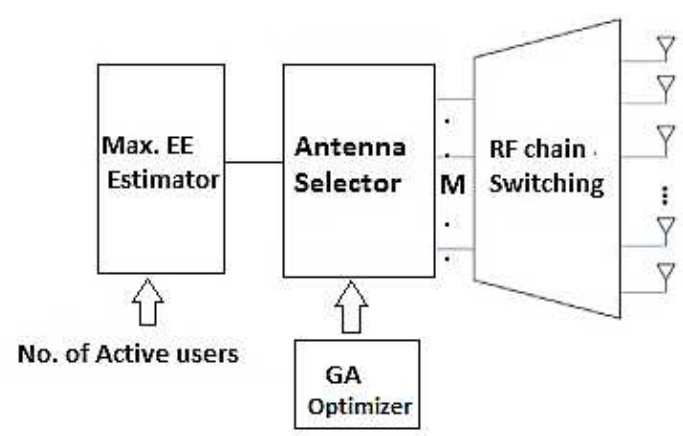

Fig.2. Block diagram of the proposed adaptive antenna selection system

The block diagram of the proposed system is depicted in Figure (2). The Maximum Energy Efficiency Estimator block is firstly supplied by the number of active users [18, 19] served by the cell [20] using the base station database or using spectrum sensing techniques [18, 22]. Then, equation (4) is used to estimate Max. EE values for different M antennas. Secondly, an Antenna selector-based Genetic optimizer is initiated to evaluate the optimum number of active antennas according to the Genetic algorithm optimization technique.
Finally, the RF chain switch operates the preevaluated number of active antennas and switches off the unnecessary antennas to maximize EE.

\subsection{EE optimization based on Genetic Algorithm Artificial intelligence}

In order to get the optimum number of massive MIMO antennas corresponding to the active users served by $5 \mathrm{G}$ cell, many optimization techniques are proposed [ 21]. However, GA optimization technique is mostly enrolled in massive MIMO networks as [22]. GA technique supports selection, crossover, and mutation processes. GA's main concepts are focused on Charles Darwin's theory of evolution.

These concepts are then applied to a computational algorithm to find solutions to optimization problems with a given objective function. [23]. A chromosome is a solution to such an optimization problem. Whereas a Population is a set of chromosomes.

The algorithm starts with an initial population of abnormal chromosomes, which are then determined using the fitness function (objective function) to choose the best ones, known as Parents Élite children have genes that donate the highest fitness value and are chosen to participate in the next generation. The remaining chromosomes would then be subjected to crossover and mutation processes to produce the next generation.

Regarding this mechanism, the target of the proposed technique is to optimize the EE in M-MIMO and improve the performance of $5 \mathrm{G}$ networks, as shown in figure 3 . then, the mentioned steps are refined till the optimum value of EE (the fitness function) is obtained. The number of antennas in M-MIMO grid is adopted to achieve the optimum EE in each cell. Therefore, we can reformat the optimization problem from equation (8) as,

$$
\text { Optimize }\{\max \operatorname{EE}(\mathrm{M}, \mathrm{K})\}
$$

Subjected to (no of active users in the cell K)

GA is mainly used to get the optimization of its input objective functions. Therefore, in this work, EE and the number of users' $\mathrm{K}$ are considered. The GA optimizers are used to optimize the consumed energy per cell and improve the M-MIMO system's EE in 5G networks. 
Table 1.GA-Based Proposed Algorithm Steps.

Proposed Algorithm

1- Initialization:

Defining GA parameters:

a) Get the group of sets $\mathrm{EE}_{0}$ that represents the random initial population according to the constraints according to equation (4).

b) Generations $=0$

2- Repeat

a) Evaluates the optimum value of Energy Efficiency (Max EE) based on equation (8)

b) Select the optimum number of Antennas based on No. of active users

c) Select Parents using the stochastic uniform selection function.

d) Select Elite children.

e) Perform Gaussian mutation and scattered crossover on parents.

f) Replace the current population with children

g) Update the no. of generations: Generations $=$ Generations +1 .

3- Stop if:

a) Optimum value of Energy Efficiency (MaxEE) is reached

b) Maximum number of Generations is occurred.

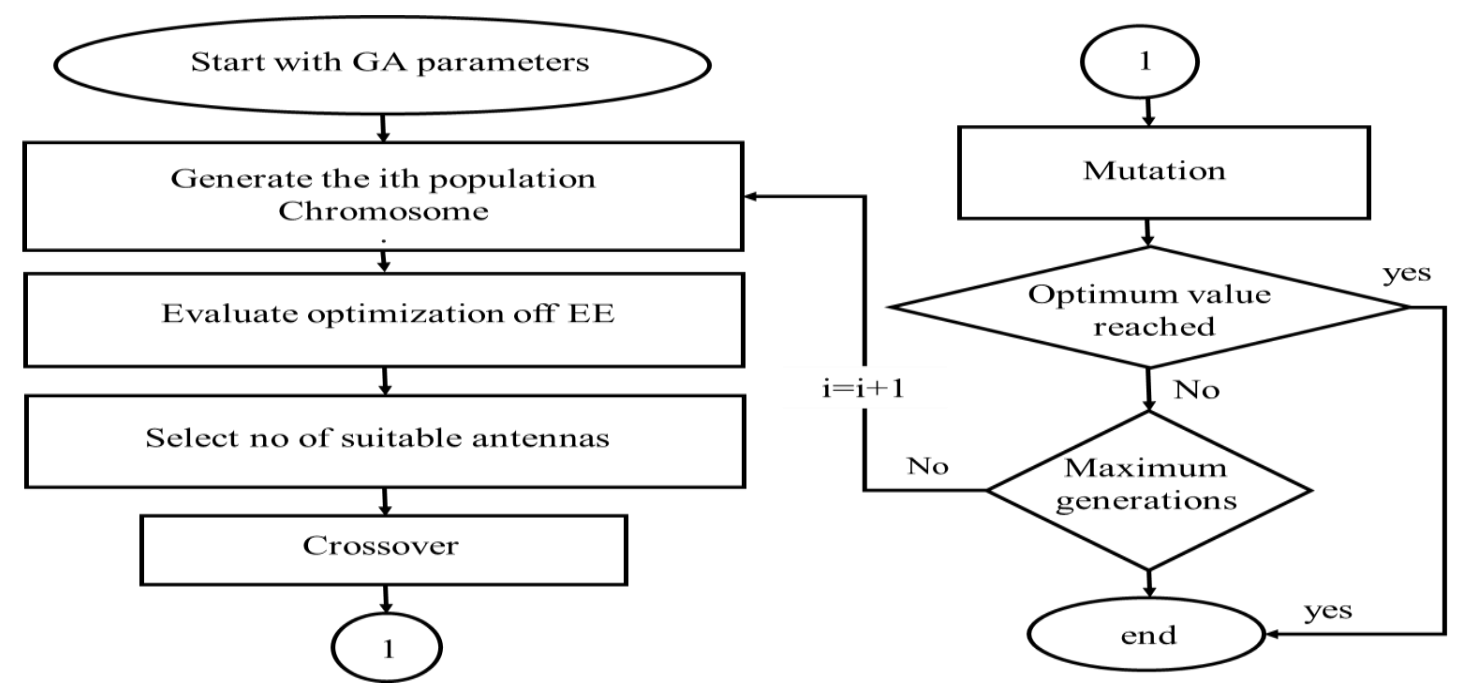

Fig.3. flow chart of the GA adaptive antenna selection system. 


\section{Simulation and Results}

Using Matlab (ver. R 2019 a) with Simulink tools, numerically how the number of UEs $(\mathrm{K})$, affects the (EE-SE) trade-off at different inter-cell interference $\left(\beta^{\prime}\right)$ values $(-3 \mathrm{db},-15 \mathrm{db})$ has been evaluated. In this scenario, EE is estimated at different numbers of users $[5,10,30$, and 40 users], the maximum EE is evaluated at each case at a constant number of active antennas where there are ten active antennas per array, under the following parameters.

Table (2). The Parameters of Simulation

\begin{tabular}{|l|l|}
\hline The Parameter & Value \\
\hline Number of Antennas, $\mathrm{M}$ & 1000 \\
\hline Bandwidth $(\mathrm{B})$ & $100 \mathrm{KHz}$ \\
\hline$(\mu)$ & 0.4 \\
\hline$P_{\mathrm{FIX}}$ & $10 \mathrm{~W}$ \\
\hline$P \mathrm{BS}$ & $1 \mathrm{~W}$ \\
\hline$P \mathrm{UE}$ & $0.5 \mathrm{~W}$ \\
\hline
\end{tabular}

Fig (3) shows the estimated EE values versus SE at various users $[5,10,30,40]$ while using ten active antennas. It is observed that the maximum $\mathrm{EE}$ value is obtained while serving ten users. Whereas for the proposed setup, the maximum value for $(K=10)$ is obtained where the sum spectral efficiency is a slowly increasing function of $K$ in case of $M=10$, while each additional UEs increases the PC by PUE $=0.5 \mathrm{~W}$. Hence, the degradation in EE for a given sum SE increases as $\mathrm{K}$ grows up.

The proposed system is considered to be adaptive by changing the number of active-antennas while the change of the number of active-users to maintain the $\mathrm{EE}$ at its maximum point (EE=MaxEE).

Table (2), shows the estimates of EE based on different number of active-antennas while serving various number of users. It is observed that the maximum, highlighted values in each column, obtained energy efficiency is increased by increasing the number of active users at increased number of active antenna.

For example, the optimum number of active antenna for serving 5 users is 20 antennas in which the (MaxEE=3.1262). Whereas, serving 10 UEs requires 30 antennas to get the (MaxEE=3.8233). Also, 30 UEs need 60 antennas to get $(\mathrm{MaxEE}=4.8691)$ and 40UEs require about 70 active-antennas to achieve (MaxEE=5.1015). From the other side, the increased number of users increases the maximum $\mathrm{EE}$ value with more active antennas.

It is observed that, EE increased by increasing the number of antennas for a constant number of users until reach the maximum value of $\mathrm{EE}$ at a suitable number of antennas before decreasing again by increasing number of active antennas as shown in Fig. (4)

When $K=10$, the EE is shown in Figure 5. for various values of the antenna-UE ratio $\mathrm{M} / \mathrm{K}$. The $\mathrm{EE}$ is an $\mathrm{M} / \mathrm{K}$ unimodal function. It rises up to $\mathrm{M} / \mathrm{K}=2$ for the investigated configuration and then gradually reduces as $\mathrm{M} / \mathrm{K}$ becomes larger. In conclusion, servicing numerous UEs while concurrently expanding the number of BS antennas (to compensate for increased interference) may enhance network EE only if the advantages and costs of installing additional RF hardware are appropriately balanced.

We looked at the EE of Massive MIMO networks in the previous section at a constant number of UEs and a variable number of BS antenna. Hence, we consider the EE from a new perspective: we build the network to achieve maximum EE, without high-priority antennas for different UEs.This is accomplished via the use of adaptive antennas and GA optimization.

According to the simulation of the GA massive MIMO scheme for an active user $(K=10)$, the optimal number of active antennas at the optimum $\mathrm{EE}$ is determined to be $(M=20)$ at the best possible performance. However, when $\mathrm{k}=20, \mathrm{M}$ is estimated to be $(\mathrm{M}=40)$. Therefore, this results is applicable as the antenna-UE ratio $\mathrm{M} / \mathrm{K}$ $=2$ as indicated in Fig. 5 .

Additionally, every change in the number of active users results in a change in the number of antennas (M) in the $5 \mathrm{G}$ cell with the maximum $\mathrm{EE}$ while varying the number of active users.

Based on the discussed simulation results, it has been proven that applying GA optimization algorithm on the proposed M-MIMO system improves the performance of the EE. However, the number of antennas in the MMIMO grid is optimized based on the number of active users in the cell, as opposed to traditional wireless networks. It has also been possible to enhance the overall efficiency and dependability of the 5G system. 


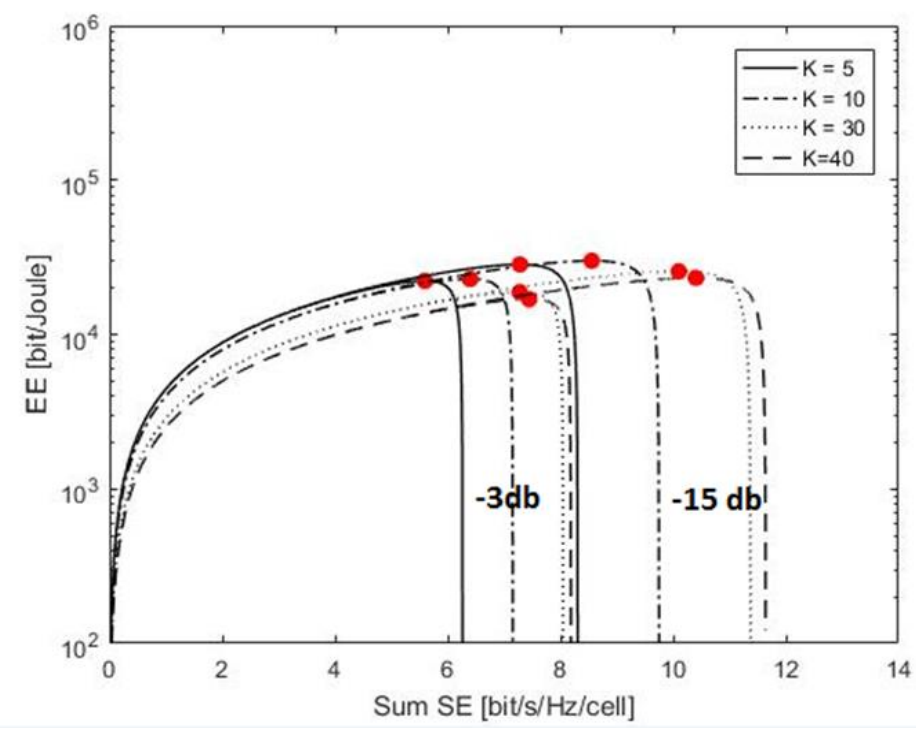

Fig. 4. The SE and EE relation for different number of K UEs at $-3 \mathrm{db},-15 \mathrm{db}$ inter-cell Interference respectively.

Table (3). The EE values for various active antennas and varied user numbers

\begin{tabular}{|c|c|c|c|c|}
\hline Users & 5 UE & 10 UE & 30 UE & 40 UE \\
\hline 10 & 2.8407 & 3.0036 & 2.5642 & 2.325 \\
\hline $\mathbf{2 0}$ & 3.1262 & 3.7374 & 3.8699 & 3.6758 \\
\hline $\mathbf{3 0}$ & 2.9914 & 3.8233 & 4.477 & 4.3954 \\
\hline $\mathbf{4 0}$ & 2.79 & 3.7228 & 4.7529 & 4.7841 \\
\hline $\mathbf{5 0}$ & 2.5924 & 3.5668 & 4.8576 & 4.9862 \\
\hline $\mathbf{6 0}$ & 2.4142 & 3.3991 & 4.8691 & 5.0777 \\
\hline $\mathbf{7 0}$ & 2.2569 & 3.2359 & 4.8281 & 5.1015 \\
\hline $\mathbf{8 0}$ & 2.1186 & 3.0828 & 4.7574 & 5.0828 \\
\hline $\mathbf{9 0}$ & 1.9968 & 2.9414 & 4.6702 & 5.0373 \\
\hline 100 & 1.8888 & 2.8116 & 4.5743 & 4.9748 \\
\hline
\end{tabular}




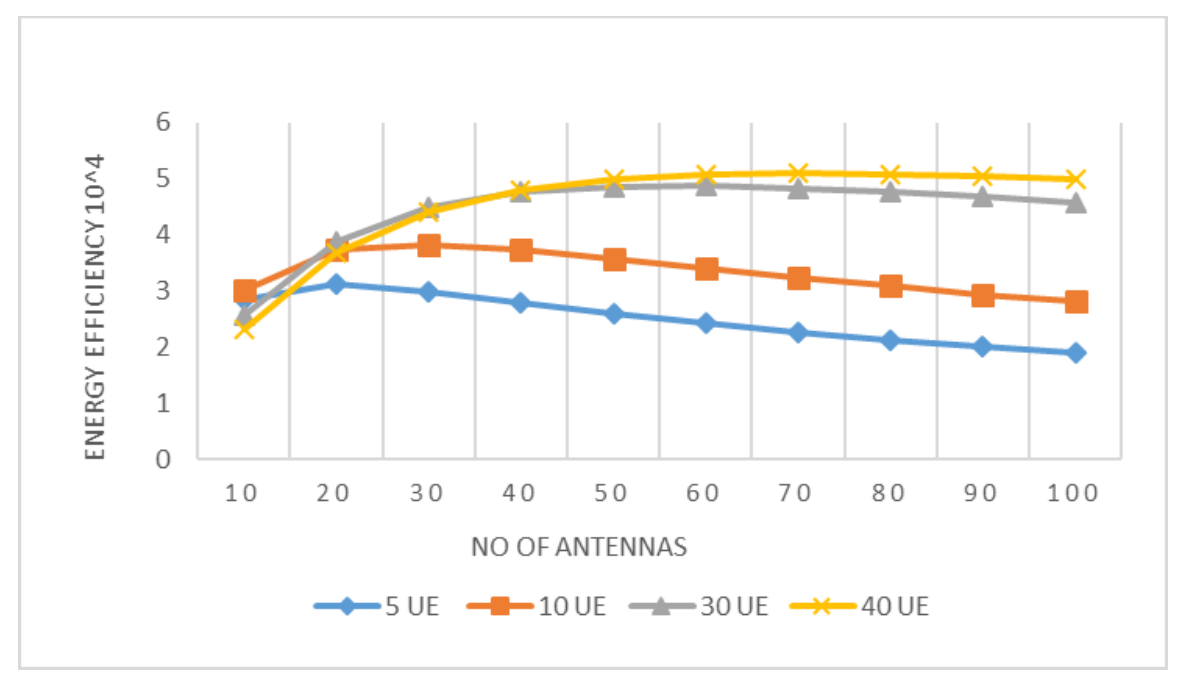

Fig. 5 EE Versus No. of antennas for different number of users

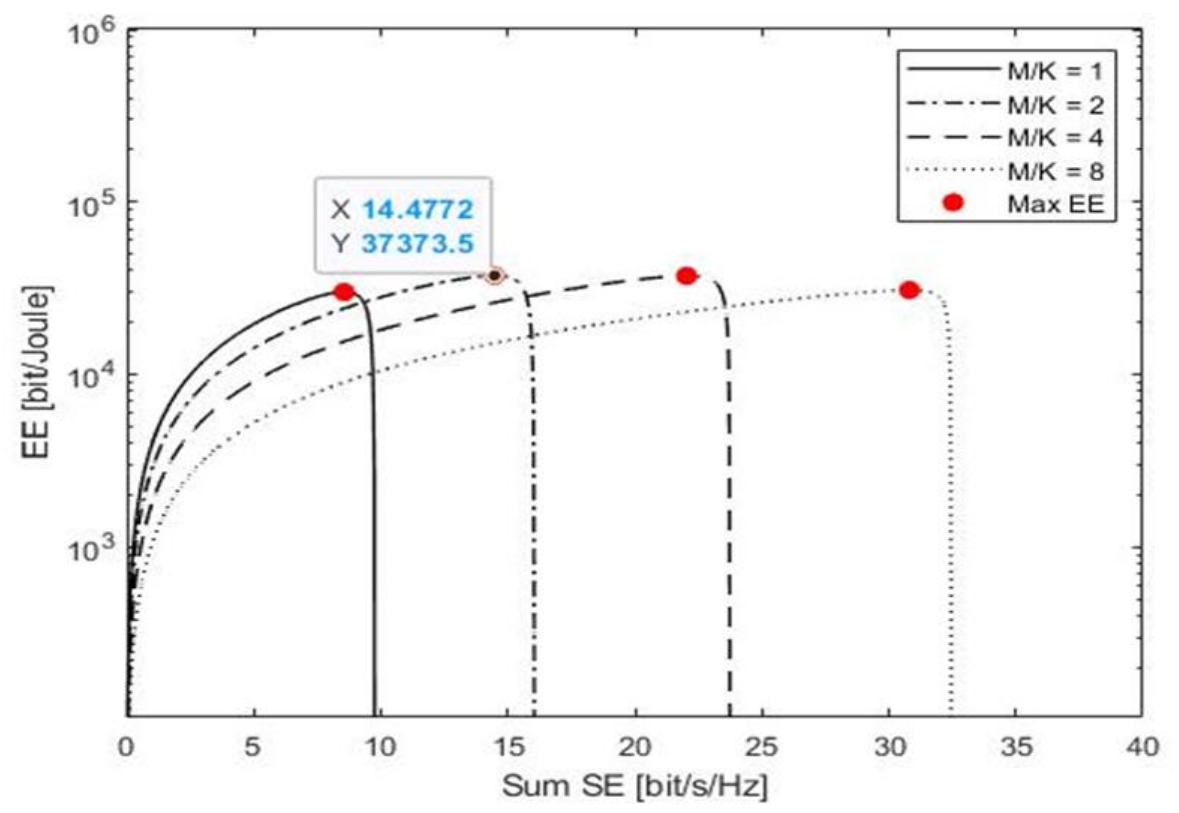

Fig.6 Sum SE and EE for various M/K antenna-UE values when $\mathrm{K}=10$ 


\section{Conclusion}

This paper proposes an approach to minimize energy consumption in a $5 \mathrm{G}$ wireless network scenario that employs highly complex antenna techniques such as massive MIMO technology. In response to changes in the number of active users within a cell, the proposed system has an adaptive number of active antennas.

The maximum energy efficiency (EEmax) obtained using GA at a given spectral efficiency has been used to estimate the number of usable antennas. The proposed system successfully saves energy and improves EE by dynamically adjusting the number of antennas to achieve maximum energy efficiency.

\section{References}

[1] J. Navarro-Ortiz, P. Romero-Diaz, S. Sendra, P. Ameigeiras, J. J. Ramos-Munoz and J. M. Lopez-Soler, "A Survey on 5G Usage Scenarios and Traffic Models," in IEEE Communications Surveys \& Tutorials, vol. 22, no. 2, pp. 905-929, Secondquarter 2020

[2] G. Gampala and C. J. Reddy, "Massive MIMO — Beyond 4G and a basis for 5G," 2018 International Applied Computational Electromagnetics Society Symposium (ACES), Denver, CO, 2018, pp. 1-2

[3] Salh, Adeeb, et al. "Trade-Off Energy and Spectral Efficiency in a Downlink Massive MIMO System." Wireless Personal Communications 106.2 (2019): 897-910.

[4] Zappone, Alessio, and Eduard Jorswieck. "Energy efficiency in wireless networks via fractional programming theory." Foundations and Trends in Communications and Information Theory 11.3-4 (2015): 185-396.

[5] Gupta, Akhil, and Rakesh Kumar Jha. "A survey of 5G network: Architecture and emerging technologies." IEEE access 3 (2015): 1206-1232.

[6] F. Rusek et al., "Scaling up MIMO: Opportunities and challenges with very large arrays," IEEE Signal Process. Mag., vol. 30, no. 1, pp. 40-60,Jan. 2013.

[7 M. Sarajli'c, L. Liu, and O. Edfors, "When are low resolution ADCs energy efficient in massive MIMO?" IEEE Access,vol.5, pp. 14837- 14853, 2017.

[8] Q. Zhang, S. Jin, K.K. Wong, Power Scaling of Uplink Massive MIMO Systems with Arbitrary-Rank Channel Means, IEEE J. Select.Topics Signal Process. 8 (2014) $966-981$. S.

[9] Gong, C. Xing, N. Yang, Y.C. Wu, Z. Fei, Energy Efficient Transmission in Multi-User MIMO Relay Channels with Perfect and Imperfect Channel State Information. IEEE Transactions Wireless Comm. 16 (2017) 38853898 .

[10] Bjornson E., Sanguinetti L., Hoydis J., Debbah M.,"Optimal Design of Energy-Efficient Multi-User
MIMO Systems: Is Massive MIMO the Answer?," IEEE Trans. Wireless Commun., vol. 14, no. 6, pp. 30593075,June 2015.

[11] W.K. Ng, E.S. Lo, R. Schober, "Energy-efficient resource allocation in OFDMA systems with large numbers of base station antennas,"IEEE Transactions on Wireless Communications 11.9 (2012): 3292-3304.

[12] W.K. Ng, E.S. Lo, R. Schober "Wireless information and power transfer: Energy efficiency optimization in OFDMA systems,"IEEE Transactions on Wireless Communications 12.12 (2013): 6352-6370

[13] Ibrahim Salah, M. Mourad Mabrook, Aziza I. Hussein, Kamel Hussein Rahouma, Comparative Study of Efficiency Enhancement Technologies in 5G Networks A survey, Procedia Computer Science,Volume 182, 2021, Pages 150-158, ISSN 1877-0509,

[14] Wyner, Aaron D. "Shannon-theoretic approach to a Gaussian cellular multiple-access channel." IEEE Transactions on Information Theory 40.6 (1994): 17131727.

[15] Xiao, Huahua, et al. "CSI feedback for massive MIMO system with dual-polarized antennas." 2015 IEEE 26th Annual International Symposium on Personal, Indoor, and Mobile Radio Communications (PIMRC). IEEE, 2015

[16] Guowang Miao, Jens Zander, Ki Won Sung, and Ben Slimane, Fundamentals of Mobile Data Networks, Cambridge University Press, ISBN 1107143217, 2016.

[17] Emil Björnson, Luca Sanguinetti, Jakob Hoydis, Merouane Debbah. Optimal Design of EnergyEfficient Multi-User MIMO Systems: Is Massive MIMO the Answer? IEEE Transactions on Wireless Communications, Institute of Electrical and Electronics Engineers, 14 (6), pp.3059-3075, 2015.

[18] M. Mourad Mabrook, Hussein A. Khalil, Aziza I. Hussein, "Artificial Intelligence Based Cooperative Spectrum Sensing Algorithm for Cognitive Radio Networks", Procedia Computer Science, Volume 163, 2019, Pages 19-29, ISSN 1877-0509,

[19] Ibrahim Salah, M. Mourad Mabrook, Aziza I. Hussein, Kamel Hussein Rahouma, Comparative Study of Efficiency Enhancement Technologies in 5G Networks A survey, Procedia Computer Science,Volume 182, 2021, Pages 150-158, ISSN 1877-0509,

[20] Shida Zhong et al. "User Oriented Transmit Antenna Selection in Massive Multi-User MIMO SDR Systems", Sensors Journal , MDPI, 2020 doi:10.3390/s20174867

[21] S. N. Sivanandam and S. N. Deepa, "Introduction to Genetic Algorithms," Computational Intelligence and Complexity, Springer, 2008.

[22] Mabrook, M.M., Taha, H.A. \& Hussein, A.I. Cooperative spectrum sensing optimization based adaptive neurofuzzy inference system (ANFIS) in cognitive radio networks. J Ambient Intell Human Comput (2020). https://doi.org/10.1007/s12652-020-02121-9

[23] H.-T. Chou and D.-Y. Cheng, "Beam-pattern calibration 
in a realistic system of phased-array antennas via the implementation of a genetic algorithm with a measurement system," IEEE Transactions on Antennas and Propagation, vol. 65, no. 2, pp. 593-601, 2017 